\title{
Statistical Description of Nonrelativistic Classical Systems
}

\author{
George H. Goedecke \\ Physics Department, New Mexico State University, Las Cruces, NM, USA \\ Email:ggoedeck@nmsu.edu
}

How to cite this paper: Goedecke, G.H. (2017) Statistical Description of Nonrelativistic Classical Systems. Journal of Modern Physics, 8, 786-802.

https://doi.org/10.4236/jmp.2017.85050

Received: March 5, 2017

Accepted: April 22, 2017

Published: April 25, 2017

Copyright $\odot 2017$ by author and Scientific Research Publishing Inc. This work is licensed under the Creative Commons Attribution International License (CC BY 4.0).

http://creativecommons.org/licenses/by/4.0/

\section{(c) (i) Open Access}

\begin{abstract}
We prove that any nonrelativistic classical system must obey a statistical wave equation that is exactly the same as the Schrödinger equation for the system, including the usual "canonical quantization" and Hamiltonian operator, provided an unknown constant is set equal to $\hbar$. We show why the two equations must have exactly the same sets of solutions, whereby this classical statistical theory (CST) and nonrelativistic quantum mechanics may differ only in their interpretations of the same quantitative results. We identify some of the different interpretations. We show that the results also imply nonrelativistic Lagrangian classical mechanics and the associated Newtonian laws of motion. We prove that the CST applied to a nonrelativistic rigid rotator yields spin angular momentum operators that obey the quantum commutation rules and allow both integer and half-odd-integer spin. We also note that the CST applied to systems of identical massive particles is mathematically equivalent to nonrelativistic quantum field theory for those particles.
\end{abstract}

\section{Keywords}

Stochastic Classical Mechanics, Stochastic Quantum Mechanics

\section{Introduction}

During the latter part of the nineteenth century, Ludwig Boltzmann initiated the application of statistics to many-particle systems. He pursued this approach despite the strongly prevailing belief in a continuum structure of matter during that period. With the introduction of the ensemble concept by Willard Gibbs in 1902, the resulting statistical mechanics achieved several great successes, including the statistical definition of entropy and derivations of thermodynamics and fluid mechanics.

During the past century, the belief that quantum mechanics (QM) is the truly fundamental theory of nature has become overwhelmingly prevailing. On the 
basis of an enormous number of correct predictions, and apparently no incorrect ones, this belief continues to strengthen despite the puzzling fact that QM makes only statistical predictions. How can a statistical theory be fundamental? Any such theory must involve a statistical treatment of some number of underlying quantities.

Many attempts have been made to establish a classical statistical foundation for the single-particle Schrödinger or Dirac equation, e.g., Bohm's hidden variable theory [1] [2]; the stochastic mechanics approach of Nelson [3] and Baublitz [4]; Okamoto's approach using a complex Langevin equation [5]; Srinivasan and Sudarshan's use of quaternion measures and the Langevin equation (to obtain the Dirac equation) [6]; use of the Fokker-Planck equation [7]; and extensive work on the global statistical hidden variable theory known as stochastic electrodynamics (SED) [7] [8] [9] [10] [11]. (The first two of these SED references are to review articles that contain references to the very many seminal and comprehensive papers on SED published during the years 1960-1990. The last three are to more recent papers on SED). Also, Gilson [12] and Collins [13] [14] [15] [16] used the Madelung transform [17] in reverse to derive a wave equation that must be obeyed by any system that satisfies a continuity equation for a non-negative density and associated flux in three-dimensional Eulidean space. Their wave equation has exactly the same form as the Schrödinger equation for a single pointlike massive spinless particle, but contains unknown functions instead of the potential energy and electromagnetic vector potential, and an unknown constant instead of Planck's constant. The unknown functions and constant would be different for, say, a classical fluid than for the statistical description of a one-particle system.

None of the abovementioned approaches has been shown to apply to all classical systems. The principal goals of the work reported in this paper are to develop a statistical description of the nonrelativistic classical motions of the coordinates of any system, based on the probability continuity relation in the coordinate configuration space, and to investigate how close is that description to the nonrelativistic quantum mechanics of the system.

We pursue those goals as follows: In section 2, we consider a general nonrelativistic classical system involving $N$ generalized (curvilinear or rectilinear) coordinates that define the $N$-dimensional configuration space (not the phase space) of the system. We note that if for any reason we wish to treat the system statistically, we may apply the continuity equation for the probability density and flux in that configuration space. Then we prove that this equation alone implies the existence of a statistical wave equation (SWE) that is exactly the same as the Schrödinger equation (SEQ) for the system, provided an unknown constant is set equal to $\hbar$ and two unknown functions are set equal to the physical potentials in the $N$-space. The new results include an actual derivation of general "canonical quantization" and the operator Hamiltonian, as well as a derivation of nonrelativistic Lagrangian classical mechanics and the associated Newton's laws of motion. Furthermore, we show that the quantitative solutions 
of the SWE must be exactly the same as those of the axiomatic SEQ for any given nonrelativistic system, but that some interpretations of these solutions must be different. In section 3, we provide analyses for three important illustrative examples, including particles with spin and systems of arbitrarily many identical particles. In sections 4 and 5, we offer a brief summary and discussion of our results, as well as a few conclusions.

\section{General Statistical Wave Equation}

In this section we treat general nonrelativistic classical systems, which are invariably described in terms of $N$ of generalized coordinates, where $N$ may be any integer $\geq 1$. For example, for a classical nonrelativistic system of $N_{p}$ identical particles, each having only translational and rigid rotational degrees of freedom, the coordinates for each particle could be three Cartesian coordinates for the center-of-mass (CM) motion, and three Euler angles for the rigid rotational motion about the $\mathrm{CM}$, or altogether $N=6 N_{p}$ generalized coordinates that comprise the configuration space of the system.

\subsection{Generalized Coordinates for Nonrelativistic Classical Systems}

We include this subsection to establish our notation, which is the notation used by Lichnerowicz [18] and Hartle [19], simply extended to $N$ dimensions. We represent the generalized-coordinate manifold of a system under consideration by the set $x=\left[x^{1}, \cdots, x^{N}\right]$, where each coordinate $x^{p}$ is an independent real continuous variable that may have any physical dimension and any range. $\mathrm{A}$ classical nonrelativistic system moves on a trajectory in this $N$-space given by $\left[x^{p}=X^{p}(t), p=(1, \cdots, N)\right]$, where $t$ is the continuous time variable as read on a reference clock.

For such systems, the kinetic energy $T$ is always a positive definite quadratic form in the coordinate velocities $\dot{X}^{p}=\mathrm{d} X^{p} / \mathrm{d} t$ :

$$
T=(1 / 2) m g_{p q}(X) \dot{X}^{p} \dot{X}^{q},
$$

where we use the extended Einstein summation convention that all repeated indices are summed over from 1 to $N$. Here, $m>0$ is an overall mass parameter that may be chosen at will, and $g_{p q}=g_{q p}$ is the metric of the $N$-space, whereby the system configuration space is a Riemannian space. In general, off-diagonal $g_{p q}$ may be nonzero, so the coordinates may not be orthogonal. We use the coordinate basis vector approach to general tensor calculus [18] [19] [20]. That approach employs the covariant (subscripted) basis vectors $\boldsymbol{e}_{p}(x)$ and the contravariant (superscripted) basis vectors $\boldsymbol{e}^{p}(x)$ as dual sets of basis vectors for the linear vector space that is tangent to the configuration space. The generalized inner or dot products of these basis vectors are symmetric and are defined by

$$
\boldsymbol{e}_{p} \cdot \boldsymbol{e}^{q}=\delta_{p}^{q} ; \quad \boldsymbol{e}_{p} \cdot \boldsymbol{e}_{q}=g_{p q} ; \quad \boldsymbol{e}^{p} \cdot \boldsymbol{e}^{q}=g^{p q},
$$

where $\delta_{p}^{q}$ is the Kronecker delta, and the matrix with elements $g^{p q}$ is the inverse of the metric matrix that has elements $g_{p q}$. Any $N$-vector field $\boldsymbol{A}(x, t)$ 
may be written as a linear combination (LC) of either set of basis vectors: $\boldsymbol{A}(x, t)=\boldsymbol{e}_{p}(x) A^{p}(x, t)=\boldsymbol{e}^{p}(x) A_{p}(x, t)$. The fields $\left(A^{p}, A_{p}\right)$ are the (contravariant, covariant) components of $\boldsymbol{A}(x, t)$. Indices may be raised or lowered with the metric: $\boldsymbol{e}^{p}(x)=g^{p q} \boldsymbol{e}_{q}(x) ; A_{p}=g_{p q} A^{q}$; etc. Please see e.g. Chap. 20 of Hartle's textbook [19], esp. Table 20.1, for more details.

\subsection{Statistical Wave Equation}

In treating classical motions statistically, one may always begin with the coordinate probability density. The fine-grained coordinate probability density in the $N$-space is

$$
\rho^{f}(x, t)=|g|^{-1 / 2} \prod_{q=1}^{N} \delta\left(x^{q}-X^{q}(t)\right),
$$

where $\delta$ is the Dirac delta and $|g|$ is the magnitude of the determinant of the metric matrix. Note that the integral of $\rho^{f}$ over all $N$-space is unity, since the volume element is $\mathrm{d} V^{N}=\sqrt{|g|} \mathrm{d} x^{1} \cdots \mathrm{d} x^{N}$. The corresponding fine-grained probability current density is $\boldsymbol{j}^{f}=\boldsymbol{e}_{p} \dot{X}^{p} \rho^{f}(x, t)$. These quantities satisfy the $N$-space continuity equation $\partial_{t} \rho^{f}+\nabla \cdot \boldsymbol{j}^{f}=0$; this equation is necessary and sufficient to guarantee conservation of probability. The $N$-space vector gradient operator $\nabla$ is defined [19] by

$$
\nabla=\boldsymbol{e}^{p}(x) \partial_{p},
$$

where $\partial_{p}=\partial / \partial x^{p}$.

These fine-grained probability densities are almost never useful in application, because it would be virtually impossible to solve for the detailed coordinate trajectories $X^{p}(t)$ even if we knew all the force fields acting on every element of the system exactly, which we do not. What is needed are smooth densities that are continuous, bounded, and at least first order differentiable. Smooth densities have always been (tacitly) assumed to exist in all statistical treatments. In earlier work [21] [22] [23] [24], we showed that the smoothing must be accomplished by an ensemble average over the global random variables associated with the classical statistical description of a system. The smooth coordinate probability density $\rho$ satisfies the requirement that $\rho(x, t) \mathrm{d} V^{N}$ be equal to the probability that the system coordinates are within the volume element $d V^{N}$ around $x$ at time $t$. Conservation of probability demands that the smooth densities $\rho$ and $\boldsymbol{j}$ must also satisfy the continuity equation

$$
\partial_{t} \rho+\nabla \cdot \boldsymbol{j}=0
$$

Note that Equation (4) must be satisfied irrespective of what stochastic process is considered, e.g., Markovian or not, and independently of what kind of stochastic dynamics is considered, e.g., the Langevin equation, the FokkerPlanck equation, etc., and independently of what kind of position-velocity or position-momentum phase space treatment may be valid. Therefore, the statistical description of a system's classical coordinates that evolves from just this continuity equation will be incomplete, but still must be obeyed. 
Now we proceed by following Collins' method [13] (which mathematically is essentially Bohm's approach in reverse order), but with our new generalization to the $N$-dimensional metric configuration space required by an arbitrary nonrelativistic classical system. The first step is to define an $N$-vector probability flow velocity field $v(x, t)$ by writing

$$
\boldsymbol{j}(x, t)=\rho(x, t) \boldsymbol{v}(x, t) .
$$

Then $\boldsymbol{v}$ is smooth since both $\rho$ and $\boldsymbol{j}$ are smooth by definition. (Note well that $v$ is not related directly to the underlying coordinate velocity components $\dot{X}^{p}(t)$; instead, it is analogous to a fluid flow velocity). This definition of $\boldsymbol{v}$ may always be made provided that $\boldsymbol{j} / \rho$ is bounded everywhere, which is the case in any physical theory. Then, with no loss of generality, one may invoke the $N$-space analog of Helmholtz' theorem to express $v$ as the sum of a gradient and another vector field that is not a gradient:

$$
\boldsymbol{v}(x, t)=\frac{\Gamma}{m} \nabla \Phi(x, t)-\boldsymbol{u}(x, t),
$$

where $\Phi(x, t)$ is an unknown real-valued function that we require to be dimensionless, whereby $\Gamma$ is an unknown real constant that has the physical dimension of angular momentum; and $\boldsymbol{u}(x, t)$ is an unknown real-valued $N$-vector field, not a gradient, having physical dimension velocity. (The methods of Gilson [12] and Collins [13] did not treat arbitrary curvilinear coordinates, and were not applied in general $N$-dimensional configuration spaces. Only Collins included the vector field $\boldsymbol{u}$, and then only in the 3-space of a single particle's CM coordinates). The next step defines the complex-valued function $\psi(x, t)$ as

$$
\psi(x, t)=\sqrt{\rho} \exp (i \Phi) .
$$

This relation is known as the Madelung transform [17]; it is usually applied to a given SEQ in a Euclidean 3-space, to obtain the Madelung/Bohm "hydrodynamical" equations, which did not include the non-gradient vector field $\boldsymbol{u}$. Here, the transform is being used in reverse in a general metric $N$-space. Note that $\rho \geq 0$, so $\sqrt{\rho}$ is well-defined and real $\geq 0$ if $\rho$ is smooth, e.g., not a product of Dirac deltas as is the fine-grained density. Equations (5)-(7) combine to yield

$$
\begin{gathered}
\rho=\psi^{*} \psi \\
\boldsymbol{j}=\frac{\Gamma}{2 i m}\left(\psi^{*} \nabla \psi-\psi \nabla \psi^{*}\right)-\mathbf{u} \psi^{*} \psi
\end{gathered}
$$

Then, requiring that the continuity Equation (4) be satisfied yields easily

$$
\frac{O \psi}{\psi}=\frac{O^{*} \psi^{*}}{\psi^{*}}=w(x, t),
$$

where $O$ is the operator

$$
O=i \Gamma \partial_{t}-\frac{1}{2 m}(-i \Gamma \nabla-m u)^{2}
$$


and $w(x, t)$ is an unknown real-valued $N$-scalar field having physical dimension energy. Therefore, the equation that must be satisfied by $\psi$ is

$$
i \Gamma \partial_{t} \psi=H^{o p} \psi
$$

where $H^{o p}$ is defined by

$$
H^{o p}=(2 m)^{-1}(-i \Gamma \nabla-m \boldsymbol{u})^{2}+w .
$$

This $N$-space SWE (12) has exactly the same form as the conventional SEQ for the system. Since the smoothed probability continuity Equation (4) is as discussed above an ensemble-averaged equation, then the SWE is an ensembleaveraged equation, and the eigenvalues of its Hamiltonian operator $H^{\text {op }}$ must be ensemble-averaged energies.

Note that we are not getting something for nothing: The quantities $w(x, t), \boldsymbol{u}(x, t), \Gamma$ and $m>0$ must be real-valued and have the physical dimensions noted above, but are otherwise arbitrary. The $N$-space probability continuity equation merely guarantees that the SWE (12) must be obeyed for arbitrary functions $w$ and $\boldsymbol{u}$. However, the meanings of $\rho$ and $\boldsymbol{j}$ are known a priori here (which was not the case during the original development and interpretation of the SEQ), whereby $\psi$ must satisfy the same continuity, integrability, boundedness, and boundary conditions that are imposed by postulate on the conventional SEQ wavefunction for a system involving an $N$-dimensional configuration space. Thus, for any system, the quantitative solutions of the SWE and SEQ must be identical, for given potentials $w$ and $\boldsymbol{u}$, if $\Gamma=\hbar$. Therefore, all typically quantum-mechanical relations, such as the uncertainty principle, also follow from the SWE, but some must be interpreted differently. We will discuss such differences in detail in later work.

Of course, one can identify $w, \boldsymbol{u}, \Gamma$, and $m$ in Equation (12) by comparison of its predictions with experimental results, or equivalently by comparison with the known SEQ. (It probably requires a complete cosmological model to predict the value of $\hbar$ ). Several authors [7] [13] have provided arguments to identify $w$ and $\boldsymbol{u}$ for a single pointlike particle. One method that has been used for such a particle, but as far as we know not in the generalized-coordinate $N$-space needed for more complex systems, is to require that Equation (12) yield the Hamilton-Jacobi equation in the classical (non-statistical) limit. This approach is quite simple. As is easily shown, (e.g. see Goedecke and Davis [25] for the 3-space version), substitution of Equation (7) into the SWE, Equation (12), yields two equations that must be satisfied. One is the probability continuity Equation (4) itself, while the other is

$$
\Gamma \partial_{t} \Phi+(2 m)^{-1}(\Gamma \nabla \Phi-m \boldsymbol{u})^{2}+w-\left(\Gamma^{2} / 2 m\right)\left(\nabla^{2} \rho^{1 / 2} / \rho^{1 / 2}\right)=0 .
$$

If $\Gamma=\hbar$, the last term on the left-hand side of this equation is the so-called quantum-mechanical potential (energy) [1] [2], generalized to the $N$-space. If it is negligible, then this equation reduces to the classical limit, the HamiltonJacobi equation for Hamilton's principal function $S=\Gamma \Phi$ for the $N$-space classical nonrelativistic system, with $w$ and $\boldsymbol{u}$ being the physical fields (elec- 
tromagnetic, gravitational, etc.) that appear in the classical $N$-space Hamiltonian of the system.

These identifications and the above derivation of the SWE from the probability continuity relation actually provide a derivation of classical mechanics (CM) for any nonrelativistic Lagrangian system as well, since the Hamilton-Jacobi equation implies the existence of a Hamiltonian, a Lagrangian containing the kinetic energy and metric (Equation (1)), the Euler-Lagrange equations, and thereby Newton's laws of motion, for arbitrarily chosen potentials $w$ and $\boldsymbol{u}$. Note that the hydrodynamic form of the SEQ, identical to Equation (14) if $\Gamma=\hbar$, does not allow quite the same conclusion, because in conventional QM the SEQ is not derived from a more fundamental equation. Also note that although Lagrangian/ Hamiltonian CM follows from Hamilton's principle, that principle is yet another postulate, in contrast to the manifestly essential conservation of probability used to derive the SWE.

Canonical quantization. We define the vector conjugate momentum operator

$$
\boldsymbol{p}^{o p}=-i \Gamma \nabla \text {. }
$$

Then the covariant components of $\boldsymbol{p}^{o p}$ follow from Equation (3):

$$
p_{r}^{o p}=\boldsymbol{e}_{r} \cdot \boldsymbol{p}^{o p}=-i \Gamma \partial_{r},
$$

and the Hamiltonian operator of Equation (13) is

$$
H^{o p}=\frac{1}{2 m}\left(\boldsymbol{p}^{o p}-m \boldsymbol{u}\right)^{2}+w .
$$

Therefore, the general rule for obtaining the SWE for any nonrelativistic classical Lagrangian system is simply to write down the classical Hamiltonian and then replace the conjugate momentum $N$-vector $\boldsymbol{P}$ by $\boldsymbol{p}^{o p}$. This is exactly the standard "canonical quantization" rule, except for the unknown constant $\Gamma$ replacing $\hbar$. Note that the commutator $\left[x^{s}, p_{r}^{o p}\right]=i \Gamma \delta_{r}^{s}$ is predicted. Also note that the presence of the combination $\left(\boldsymbol{p}^{o p}-m \boldsymbol{u}\right)$ in $H^{o p}$ ensures that the $N$-vector potential field $\boldsymbol{u}$ must have the same significance as the electromagnetic vector potential for a single electric monopole, that of a gauge field.

\section{Examples}

In this section, we consider three examples that should help clarify the generalized-coordinate approach. One is a system of two spinless pointlike particles that may have different masses. Another is a system of arbitrarily many identical spinless pointlike particles with two-body central force instantaneous internal interactions. A third is a system of one nonrelativistic rigid rotator. In these examples, we put $\Gamma=\hbar$, as implied by the general results above.

\subsection{Two Pointlike Particles}

The designation "pointlike" does not mean that the particles are actual points; instead, it means that the model particles considered are allowed no coordinates 
other than their CM coordinates. In this example, let the masses be $m_{1}, m_{2}$, and choose three Cartesian coordinates for each particle's CM location, $x^{1}, x^{2}, x^{3}$ for particle $1, x^{4}, x^{5}, x^{6}$ for particle 2. Then the kinetic energy $T$ on the trajectory $\left(x^{p}=X^{p}(t), p=1, \cdots, 6\right)$ is

$$
T=\frac{1}{2} m\left\{\frac{m_{1}}{m} \dot{X}^{i} \dot{X}^{i}+\frac{m_{2}}{m} \dot{X}^{i+3} \dot{X}^{i+3}\right\}=\frac{1}{2} m g_{p q} \dot{X}^{p} \dot{X}^{q},
$$

where the index $i$ ranges and sums from 1 to 3. From Equation (18), we may read off the diagonal 6-space metric:

$$
g_{11}=g_{22}=g_{33}=m_{1} / m ; \quad g_{44}=g_{55}=g_{66}=m_{2} / m,
$$

with other components zero. Since $g^{p q}=\boldsymbol{e}^{p} \cdot \boldsymbol{e}^{q}$ in general, and $g^{p q}=1 / g_{p q}$ for $p=q$ in this example, and zero otherwise, we have

$$
\boldsymbol{e}^{p}=\sqrt{m / m_{1}} \hat{\boldsymbol{e}}_{i}, p=i ; \quad \boldsymbol{e}^{p}=\sqrt{m / m_{2}} \hat{\boldsymbol{e}}_{i+3}, p=i+3,
$$

where $\hat{\boldsymbol{e}}_{i}$ and $\hat{\boldsymbol{e}}_{i+3}$ are the Cartesian unit basis vectors in the 6-space.

For this example, we consider the unperturbed central force case, by choosing $\boldsymbol{u}=0$ and $w=w(r)_{x=X(t)}$ in the classical Hamiltonian, where

$$
r=\left[\left(x^{i}-x^{i+3}\right)\left(x^{i}-x^{i+3}\right)\right]^{1 / 2}
$$

is the distance between the particle CM's. The classical Hamiltonian is $H=\boldsymbol{P}^{2} / 2 m+w(r)_{x=X(t)}$, and $\boldsymbol{p}^{o p}=-i \hbar \nabla=-i \hbar \boldsymbol{e}^{p} \partial_{p}$. Thus, according to our general results in Section 2, the Hamiltonian operator in the statistical wave equation is $H^{o p}=\left(-\hbar^{2} / 2 m\right) \nabla^{2}+w(r)$. Therefore, that SWE is

$$
i \hbar \partial_{t} \psi=-\left(\hbar^{2} / 2 m_{1}\right) \partial_{i} \partial_{i} \psi-\left(\hbar^{2} / 2 m_{2}\right) \partial_{i+3} \partial_{i+3} \psi+w \psi
$$

At this point, one may go to the conventional notation $x^{i}=x_{1}^{i}, x^{i+3}=x_{2}^{i}$, and then to the system CM and relative coordinates.

One reason for choosing this particular example is that it is probably the simplest two-particle example of the general method derived in section 2 . Another reason is to emphasize that what you get in the Hamiltonian operator in the derived SWE is exactly what you have included in the classical Hamiltonian. For example, it is clearly physically incorrect to choose $\boldsymbol{u}=0$ and thus omit all incident and self radiation fields. It is fortunate that perturbation theory works well in some cases. It is also incorrect in principle to neglect retardation in two-body interactions, but that will be a negligible effect in cases involving slow motions of particles that remain close together.

\subsection{Many Identical Pointlike Particles}

Consider the extension of the two-particle system above to $N_{p}$ pointlike spinless particles interacting with each other via two-body central force potential energies involving their CM coordinates and also allowing external electromagnetic fields. We let the particles be identical, each with electric charge $q$, mass $m$, and possibly other charges, and each with CM location but no other degrees of freedom. Then the classical nonrelativistic Lagrangian, Hamiltonian, and motion equations each involve $N=3 N_{p}$ coordinates, 
$x^{p}=X^{p}(t), p=1, \cdots, N$. The development in Section 2 yields the general statistical wave Equation (12) involving these $N$ coordinates. For this example, the metric may be chosen as the $N$-space Kronecker delta metric, $g_{p q}=\delta_{p q}=g^{p q}$, corresponding to three independent Cartesian coordinates for each particle CM. In order to achieve a familiar notation, we relabel the coordinates by letting $\left(x^{p}, p=1, N\right) \rightarrow\left(x_{n}^{i}, n=1, N_{p}, i=1,3\right)$, so that $n$ is a particle index and $i$ is a Cartesian coordinate index. Then, by analogy with the previous example, the simplest nontrivial unperturbed classical Hamiltonian contains $\boldsymbol{u}=0$ and

$$
w(x, t)=w^{\mathrm{int}}=\frac{1}{2} \sum_{n=1}^{N_{p}} \sum_{n^{\prime}=1}^{N_{p}} V\left(r_{n n^{\prime}}\right),
$$

where terms with $n=n^{\prime}$ are omitted from the double sum,

$r_{n n^{\prime}}=\left[\left(x_{n}^{i}-x_{n^{\prime}}^{i}\right)\left(x_{n}^{i}-x_{n^{\prime}}^{i}\right)\right]^{1 / 2}$, and $V$ is a two-body central force interaction energy that could involve not only the Coulomb repulsion but also other forces such as Yukawa interactions and gravity. If we allow given external electromagnetic potentials $\varphi^{\mathrm{ext}}, A_{i}^{\mathrm{ext}}$ to perturb the system, then the Hamiltonian would include the terms

$$
w(x, t)=w^{\mathrm{int}}+q \sum_{n=1}^{N_{p}} \varphi^{\mathrm{ext}}\left(x_{n}, t\right) ; \quad u_{n, i}(x, t)=(q / m c) A_{i}^{\mathrm{ext}}\left(x_{n}, t\right),
$$

where $x_{n}$ stands for $\left(x_{n}^{1}, x_{n}^{2}, x_{n}^{3}\right)$. The $N$-vector $\boldsymbol{u}=\boldsymbol{e}^{p} u_{p}=\boldsymbol{e}_{n}^{i} u_{n, i}$, where $\boldsymbol{e}_{n}^{i}=\hat{\boldsymbol{e}}^{i}$, the Cartesian unit basis vector, the same for all $n$. Again we emphasize that the functions $w$ and $\boldsymbol{u}$ that appear in the Hamiltonian operator are exactly those that are chosen for inclusion in the classical Hamiltonian. This often-used example omits internal vector potentials and also neglects retardation and self-fields.

For the identical particles in this example, the total Hamiltonian is invariant under all pair interchanges of particle indices. This invariance leads immediately to the result that the total wavefunction solution of the general many-particle SWE must either change sign under each pair interchange, yielding Fermions, or not change sign, yielding Bosons. As discussed in detail by Schweber [26], the set of all Schrödinger equations for $N_{p}=(1,2,3, \cdots, \infty)$ identical particles is mathematically equivalent to the "second quantized" many-particle quantum field theory for Fermions or Bosons in occupation number space.

\subsection{Nonrelativistic Rigid Rotator}

Many authors have considered classical spinning top models and their possible connections to quantum spin and magnetic moment, e.g. [27]-[37]. Their treatments either postulate the usual commutation rules for the Cartesian components of the spin operator in the non-rotating coordinate system, by analogy with the rules for orbital angular momentum, or postulate that the momenta conjugate to the Euler angles become operators equal to $-i \hbar$ times derivatives with respect to to the angles, by analogy with conjugate translational momenta. In our approach, no such postulates are needed; we simply apply the 
general statistical description developed in section 2 to the nonrelativistic rotations of a rigid body described by three Euler angle coordinates, as shown below.

\subsubsection{Euler Angles and Angular Velocity}

We include this subsection to establish our notation and method. For a rigid rotator with a fixed CM, the coordinates are a set of Euler angles, $\left(\alpha^{b}=x^{b}, b=1,2,3\right)$. We adopt the set $\left(\alpha^{b}=\alpha, \beta, \gamma\right)$ used by Arfken [38], and called the "zyz" set [39]. (Altogether there are twelve sets of Euler angles; all yield the same general results found below). A general rotation of Cartesian coordinates from a non-rotating system with Cartesian unit basis vectors $\hat{\boldsymbol{e}}_{i}$ to a rotating system with Cartesian unit basis vectors $\hat{\overline{\boldsymbol{e}}}_{i}$, with the origins of both systems at the body center of mass, is obtained by the relation

$$
\hat{\overline{\boldsymbol{e}}}_{i}=R_{i j}^{z}\left(\alpha^{3}\right) R_{j k}^{y}\left(\alpha^{2}\right) R_{k l}^{z}\left(\alpha^{1}\right) \hat{\boldsymbol{e}}_{l}=R_{i l}(\alpha) \hat{\boldsymbol{e}}_{l},
$$

where the orthogonal matrices $R^{z}$ and $R^{y}$ are given by

$$
R^{z}(\mu)=\left(\begin{array}{ccc}
\cos \mu & \sin \mu & 0 \\
-\sin \mu & \cos \mu & 0 \\
0 & 0 & 1
\end{array}\right) ; \quad R^{y}(\mu)=\left(\begin{array}{ccc}
\cos \mu & 0 & -\sin \mu \\
0 & 1 & 0 \\
\sin \mu & 0 & \cos \mu
\end{array}\right) .
$$

Thus, the complete rotation is specified by the orthogonal matrix $R(\alpha)=R^{z}\left(\alpha^{3}\right) R^{y}\left(\alpha^{2}\right) R^{z}\left(\alpha^{1}\right)$.

The angular velocity 3 -vector can be found from the relations defining rigidly rotating Cartesian coordinates,

$$
\mathrm{d} \hat{\overline{\boldsymbol{e}_{i}}} / \mathrm{d} t=\omega \times \hat{\overline{\boldsymbol{e}_{i}}}
$$

where $x$ is the cross-product, and $\omega$ is the instantaneous angular velocity. Its Cartesian components $\omega_{i}=\hat{\boldsymbol{e}}_{i} \cdot \boldsymbol{\omega}$ in the non-rotating frame, and $\bar{\omega}_{i}=\hat{\overline{\boldsymbol{e}}}_{i} \cdot \boldsymbol{\omega}$ in the rotating frame, can be obtained using Equations (24)-(26). The results are

$$
\omega_{i}=a_{i b} \dot{\alpha}^{b} ; \quad \bar{\omega}_{i}=b_{i b} \dot{\alpha}^{b}=R_{i k} \omega_{k},
$$

where we have specified the time-dependent trajectories of the Euler angles by $\alpha^{b}=\alpha^{b}(t)$. The matrices $(a)$ and $(b)=(R)(a)$ are easily shown to be

$$
(a)=\left(\begin{array}{ccc}
0 & -\sin \alpha^{1} & \sin \alpha^{2} \cos \alpha^{1} \\
0 & \cos \alpha^{1} & \sin \alpha^{2} \sin \alpha^{1} \\
1 & 0 & \cos \alpha^{2}
\end{array}\right) ; \quad(b)=\left(\begin{array}{ccc}
-\sin \alpha^{2} \cos \alpha^{3} & \sin \alpha^{3} & 0 \\
\sin \alpha^{2} \sin \alpha^{3} & \cos \alpha^{3} & 0 \\
\cos \alpha^{2} & 0 & 1
\end{array}\right) \text {. }
$$

\subsubsection{Model Rotator, Metric, and Conjugate Momenta}

In this introductory work, we treat a very simple model rotator, a non-translating but freely rotating rigid extended symmetric object having only the attributes of mass $m$, three equal principal moments of inertia $I$, and three Euler angle coordinates and the associated 3-vector angular velocity $\omega(t)$ about the fixed CM. The Lagrangian $L$ is the kinetic energy

$$
L=T=\frac{1}{2} I \omega^{2}=\frac{1}{2} I a_{i b} a_{i c} \dot{\alpha}^{b} \dot{\alpha}^{c}=\frac{1}{2} I b_{i b} b_{i c} \dot{\alpha}^{b} \dot{\alpha}^{c} .
$$

The metric of the Euler angle 3-space is easily identified as 
$g_{b c}=(I / m) a_{i b} a_{i c}=(I / m) b_{i b} b_{i c}$. It is not diagonal, so these Euler angle coordinates are not orthogonal. We do not need the metric in what follows.

We may define the conjugate momentum 3-vector by

$$
\boldsymbol{S}=\partial L / \partial \boldsymbol{\omega}=I \boldsymbol{\omega},
$$

where $S$ is the intrinsic (spin) angular momentum about the CM, conjugate to $\boldsymbol{\omega}$. Then the Hamiltonian $H=T$ is simply

$$
H=\frac{1}{2 I}(S)^{2} .
$$

The (angular) momenta conjugate to the angles are

$$
P_{b}=\partial L / \partial \dot{\alpha}^{b}=I a_{i b} a_{i c} \dot{\alpha}^{c}=I b_{i b} b_{i c} \dot{\alpha}^{c} .
$$

Contraction with $a_{b k}^{-1}$ and $b_{b k}^{-1}$ and comparison with Equation (30) yields

$$
S_{k}=a_{b k}^{-1} P_{b}=I \omega_{k} ; \bar{S}_{k}=b_{b k}^{-1} P_{b}=I \bar{\omega}_{k},
$$

where the $\left(S_{k}, \bar{S}_{k}\right)$ are the (non-rotating system, rotating system) Cartesian components of the conjugate spin angular momentum 3-vector $\boldsymbol{S}$.

\subsubsection{Statistical Wave Equation and Spin Operators}

The relevant SWE for any nonrelativistic system having three coordinates is the three-dimensional version of the general SWE (12). In this case, the fields $\boldsymbol{u}$ and $w$ are zero, and the gradient operator in the Euler angle space is given by

$$
\nabla=\boldsymbol{e}^{b} \partial_{b},
$$

where $\partial_{b}=\partial / \partial \alpha^{b}$. Therefore, the conjugate classical momenta must be replaced by the operators

$$
P_{b} \rightarrow p_{b}^{o p}=-i \hbar \partial_{b},
$$

just as postulated by some of the authors mentioned above. Writing Equation (31) in summation notation and making these substitutions yields

$$
H^{o p}=\frac{1}{2 I} S_{i}^{o p} S_{i}^{o p}
$$

where, from Equations (33) and (35),

$$
S_{i}^{o p}=a_{b i}^{-1} p_{b}^{o p}=-i \hbar a_{b i}^{-1} \partial_{b} .
$$

It is not difficult to show that the operators $H^{o p}$ and $S^{o p}$ are Hermitian provided the coordinate probability density obeys periodic boundary conditions in the Euler angle space. Using Equations (28) and (37), it is easy to show that the spin operators $S_{i}^{o p}$ satisfy the usual angular momentum commutation rules,

$$
\left[S_{i}^{o p}, S_{j}^{o p}\right]=i \hbar \epsilon_{i j k} S_{k}^{o p},
$$

where $\epsilon_{i j k}$ is the completely antisymmetric Levi-Civita symbol. Equations (33) and (35) also yield the expressions

$$
\bar{S}_{i}^{o p}=b_{b i}^{-1} p_{b}^{o p}=-i \hbar b_{b i}^{-1} \partial_{b} .
$$

Equations (28) and (39) then yield easily 


$$
\left[\bar{S}_{i}^{o p}, \bar{S}_{j}^{o p}\right]=-i \hbar \epsilon_{i j k} \bar{S}_{k}^{o p}
$$

Note the minus sign, compared to Equation (38). These "left-handed" commutation rules must be obeyed by the rotating system Cartesian components of $S$.

Note that $H^{o p}$, Equation (31), has eigenvalues $E_{s}=s(s+1) \hbar^{2} / 2 I$, where the spin $s=0,1 / 2,1,3 / 2,2,5 / 2, \cdots, \infty$. Therefore, the energy required to produce a transition from $s=1 / 2$ to $s=3 / 2$ is $\Delta E=3 \hbar^{2} / 2 I$. Let $I=m a^{2}$, where $a$ is the approximate linear extension of the model rotator. For a nucleon, with $m \approx 10^{3} \mathrm{MeV} / \mathrm{c}^{2}$ and $a \approx 1 \mathrm{fm}, \Delta E \approx 50 \mathrm{MeV}$, so (unstable) spin-3/2 baryons should exist, and they do. However, for an electron, with $m \approx 0.5 \mathrm{MeV} / \mathrm{c}^{2}$ and $a \leq 10^{-5} \mathrm{fm}, \Delta E \geq 10^{15} \mathrm{MeV}$. One must conclude that creating a spin-3/2 lepton having $a \leq 10^{-5} \mathrm{fm}$ is virtually impossible. Furthermore, any model of a charged object having semi-definite charge density, mass and intrinsic magnetic moment of the order of electronic values, and relevant linear extension $a \leq 10^{-5} \mathrm{fm}$, rotating rigidly with angular speed $\omega$, and having spin angular momentum of order $\hbar$, predicts a linear surface speed $\omega a \gg c$ [40]. Therefore, rotations and translations of electrons and probably other leptons must be treated relativistically, which is beyond the scope of this work. (See the Appendix for a simple illustrative relativistic rotator model).

In a paper to follow, we will derive several relevant detailed results for a charged nonrelativistically spinning top of arbitrary shape and structure immersed in a magnetic field, including the following: 1) The commutation rules of Equations (38) and (40) are unchanged, whereby the commuting operators $\left(\boldsymbol{S}^{o p}\right)^{2}, S_{3}^{o p}, \bar{S}_{3}^{o p}$ still have the same simultaneous eigenfunctions, sometimes called the Wigner harmonics, with eigenvalues $s(s+1) \hbar^{2}, m_{s} \hbar, \bar{m}_{s} \hbar$, respectively, where $-s \leq m_{s} \leq s$, and independently, $-s \leq \bar{m}_{s} \leq s$, in integer steps; 2) a general rigid rotator wavefunction may be a superposition of integer spin eigenstates only, or of half-odd-integer spin eigenstates only, but not both, whereby e.g. electromagnetic transitions from a half-odd-integer spin state to an integer spin state are forbidden; 3) In the presence of a constant uniform magnetic field, one obtains the expected spinor equations consisting of $2 s+1$ coupled equations for each choice of $s$ and $\bar{m}_{s}$. For example, for $s=1 / 2$, and $\bar{m}_{s}$ either $1 / 2$ or $-1 / 2$, one obtains two coupled equations that comprise the Pauli-Schrödinger equation, when written in matrix/spinor form; and 4) For an anisotropic rotator, the Hamiltonian eigenvalues may depend on $m_{s}$ and/or $\bar{m}_{s}$ as well as on $s$.

\section{Summary, Discussion, and Conclusions}

In this work, using a derivation from first principles with no approximations, we proved that any nonrelativistic classical physical system must obey a statistical wave equation (SWE) that has the same form and the same quantitative solutions as the Schrödinger equation (SEQ) for the system. In the non-statistical ("classical") limit, the SWE yields a system Hamiltonian and Lagrangian and thus the Euler-Lagrange equations and Newton's laws of motion for the system 
coordinates. The SWE also yields quantum spin and many-body quantum field theory for nonrelativistic systems. On the basis of these results, should we not conclude that the classical statistical theory (CST) developed in this work actually provides a derivation of nonrelativistic classical and quantum dynamics for all Lagrangian systems composed of massive particles?

Our answer to that question is "not yet, and maybe never". In earlier work [21] [22] [23] [24] and in this work (see Section 2.2) we found that a classical statistical theory based on continuity equations does not allow the conventional QM interpretations of the same quantitative results. In particular, the SWE by itself does not provide a complete statistical description of any system. For example, restrictions on initial conditions may exist such that the system cannot be in a single excited state initially, but only in a mixture of excited states [23] [24]. Also, the SWE is an ensemble-averaged equation, so its quantized Hamiltonian eigenvalues, the same as those of the SEQ, are ensemble-averaged energies; the actual energies are not quantized. Nevertheless, standard perturbation theory applied to a perturbed SWE still yields the Einstein rule involving the differences of the unperturbed eigenvalues for the frequencies of the dominant transitions, as well as the conventional transition rules and probabilities. (Prediction of transition probabilities for spontaneous emission using either the SEQ or the SWE requires either quantized electromagnetic fields or the inclusion of a radiation reaction vector portential in the vector potential $\boldsymbol{u}$; please see below, and also see reference [24] for a detailed discussion of this feature).

Detailed analysis of such profound interpretational differences is well beyond the scope of this introductory paper. Our results would require an ensemble interpretation of $\mathrm{QM}$, as well as other re-interpretations; see e.g. work by Ballentine [41] [42]. Furthermore, all our derivations follow conventional logical paths, but in reverse. For example, in this work we started our derivation with classical systems that do have coordinate trajectories, and used the Madelung transform in reverse to obtain the SWE/SEQ. On the other hand, Erwin Schrödinger inferred his SEQ nearly a century ago; the Madelung transform was applied to the SEQ to obtain its full hydrodynamic form, Equation (14). However, scientists are still discussing whether classical trajectories are compatible with conventional QM [43].

The CST derived herein makes sense only if there are reasons why even a one-particle classical system might require a statistical treatment. One possible reason is the known fact that any classical system is continually bombarded by thermal and other highly fluctuating background radiation fields. One model background field used in Stochastic Electrodynamics [7] [8] is the electromagnetic stochastic zero-point field (SZPF), which is a stochastic analog of the quantized electromagnetic field; see e.g. [21]. Up to a multiplicative adjustable constant, chosen to be $\hbar$, the SZPF is the only stochastic electromagnetic free field having a rotation-invariant, translation-invariant, and Lorentz-invariant ensemble-averaged energy density spectrum. Postulating the presence of this 
field enabled Boyer [44] to derive the Planck spectrum without quantum assumptions, and also allowed classical statistical derivations of Casimir forces [45]. The presence of the SZPF also yields a phase space derivation that automatically includes a radiation reaction vector potential in the SWE/SEQ, as well as a companion equation that restricts initial conditions, for the nonrelativistic charged harmonic oscillator [23] [24]. Together, these equations predict the correct $(\mathrm{QM})$ transition rates, including those for spontaneous emission, without quantization of classical electromagnetic fields. The presence of the SZPF also provides a physical rationale for the interference phenomena predicted by the SWE/SEQ. All of these SED results are based on ensemble averages over the random amplitudes of the plane waves that comprise the SZPF.

\section{Conclusion}

In conclusion: Before we are willing to conclude that the CST actually provides a derivation of a good part of modern physics, we feel that at least two things must be accomplished. One is a statistical treatment of relativistic classical systems, with a closer correspondence to our nonrelativistic treatment herein than our work reported in 2010 [25]. The other is a resolution of the interpretational differences between the CST and conventional QM. This latter accomplishment may require clever experiments that will take some time.

\section{Acknowledgements}

The author would like to thank Stephen Pate, Michael Engelhardt, and Stefan Zollner for helpful discussions and assistance in preparing the manuscript.

\section{References}

[1] Bohm, D. (1952) Physical Review, 85, 166. https://doi.org/10.1103/PhysRev.85.166

[2] Bohm, D. (1952) Physical Review, 85, 180. https://doi.org/10.1103/PhysRev.85.180

[3] Nelson, E. (1966) Physical Review, 150, 1079. https://doi.org/10.1103/PhysRev.150.1079

[4] Baublitz Jr., M. (1988) Progress of Theoretical Physics, 80, 232. https://doi.org/10.1143/PTP.80.232

[5] Okamoto, H. (1990) Journal of Physics A: Mathematical and General, 23, 5535. https://doi.org/10.1088/0305-4470/23/23/025

[6] Srinivasan, S. and Sudarshan, E. (1996) Journal of Physics A: Mathematical and General, 29, 5181. https://doi.org/10.1088/0305-4470/29/16/034

[7] Boyer, T. (1980) A Brief Survey of Stochastic Electrodynamics. In: Barut, A.O., Ed., Foundations of Radiation Theory and Quantum Electrodynamics, Plenum, NY, 49-63. https://doi.org/10.1007/978-1-4757-0671-0_5

[8] de la Pena, L. and Cetto, A. (1996) The Quantum Dice: An Introduction to Stochastic Electrodynamics. Kluwer, The Netherlands. https://doi.org/10.1007/978-94-015-8723-5

[9] Manfredi, G., Mola, S. and Faix, M.R. (1993) European Journal of Physics, 14, 101. https://doi.org/10.1088/0143-0807/14/3/002

[10] Dechoum, K. and Frana, H. (1995) Foundations of Physics, 25, 1599-1620. 
https://doi.org/10.1007/BF02055510

[11] Dechoum, K., Frana, H. and Malta, C. (1998) Physics Letters A, 24, 93-102.

[12] Gilson, J. (1968) Mathematical Proceedings of the Cambridge Philosophical Society, 64, 1061-1070. https://doi.org/10.1017/S0305004100043826

[13] Collins, R. (1977) Lettere al Nuovo Cimento (1971-1985), 18, 581-584. https://doi.org/10.1007/BF02785113

[14] Collins, R. (1977) Foundations of Physics, 7, 475-494. https://doi.org/10.1007/BF00708864

[15] Collins, R. (1979) Lettere al Nuovo Cimento (1971-1985), 25, 473-475. https://doi.org/10.1007/BF02776336

[16] Collins, R. (1992) Foundations of Physics Letters, 5, 63-69. https://doi.org/10.1007/BF00689797

[17] Madelung, E. (1926) Zeitschrift für Physik, 40, 322-326. https://doi.org/10.1007/BF01400372

[18] Lichnerowicz, A. (1962) Elements of Tensor Calculus. Methuen, London.

[19] Hartle, J. (2003) Gravity. Addison-Wesley, Reading, MA.

[20] Goedecke, G. (2011) The European Physical Journal Plus, 126, 32. https://doi.org/10.1140/epjp/i2011-11032-x

[21] Goedecke, G. (1983) Foundations of Physics, 13, 1101-1119. https://doi.org/10.1007/BF00728139

[22] Goedecke, G. (1983) Foundations of Physics, 13, 1121-1138. https://doi.org/10.1007/BF00728140

[23] Goedecke, G. (1983) Foundations of Physics, 13, 1195-1220. https://doi.org/10.1007/BF00727993

[24] Goedecke, G. (1984) Foundations of Physics, 14, 41-63. https://doi.org/10.1007/BF00741646

[25] Goedecke, G. and Davis, B. (2010) Nuovo Cimento B, 125, 941-955.

[26] Schweber, S. (1961) An Introduction to Relativistic Quantum Field Theory. Chap. 6, Row, Peterson, Evanston, IL.

[27] Bopp, F. and Haag, R. (1950) Zeitschrift für Naturforschung, 5A, 644.

[28] Nyborg, P. (1962) Il Nuovo Cimento (1955-1965), 23, 47. https://doi.org/10.1007/BF02733541

[29] Corben, H.C. (1968) Classical and Quantum Theories of Spinning Particles. Holden-Day, San Francisco.

[30] de la Peña-Auerbach, L. (1971) Journal of Mathematical Physics, 12, 453. https://doi.org/10.1063/1.1665609

[31] Young, R. (1976) American Journal of Physics, 44, 581. https://doi.org/10.1119/1.10383

[32] Barut, A. and Bracken, A. (1981) Physical Review D, 23, 2454. https://doi.org/10.1103/PhysRevD.23.2454

[33] Barut, A. and Bracken, A. (1981) Physical Review D, 24, 3333. https://doi.org/10.1103/PhysRevD.24.3333

[34] Jáuregi, R. and de la Peña, L. (1981) Physics Letters A, 86, 280-282.

[35] Barut, A. and Zanghi, N. (1984) Physical Review Letters, 52, 2009. https://doi.org/10.1103/PhysRevLett.52.2009

[36] Barut, A., Božić, M. and Marić, Z. (1992) Annals of Physics, 214, 53-83. 
[37] Arsenovic, D., Barut, A., Marić, Z. and Božić, M. (1995) Il Nuovo Cimento B (1971-1996), 110, 163-175. https://doi.org/10.1007/BF02741499

[38] G. Arfken (1985) Mathematical Methods for Physicists. 3rd Edition, Academic Press, NY.

[39] Goldstein, H. (1980) Classical Mechanics. 2nd Edition, Addison-Wesley, Reading, MA.

[40] Griffiths, D. (2005) Introduction to Quantum Mechanics. 2nd Edition, PrenticeHall, London.

[41] Ballentine, L. (1970) Reviews of Modern Physics, 42, 358. https://doi.org/10.1103/RevModPhys.42.358

[42] Ballentine, L. (1998) Quantum Mechanics: A Modern Development. World Scientific, NY. https://doi.org/10.1142/3142

[43] Wechsler, S. (2017) Journal of Modern Physics, 8, 68-81. https://doi.org/10.4236/jmp.2017.81006

[44] Boyer, T. (1969) Physical Review, 182, 1374. https://doi.org/10.1103/PhysRev.182.1374

[45] Boyer, T. (1973) Physical Review A, 7, 1832. https://doi.org/10.1103/PhysRevA.7.1832 


\section{Appendix A: Relativistic Rotation}

As mentioned above, one objection to a nonrelativistic extended spinning electron model having semidefinite charge density is that it seems to require tangential linear speeds $v$ that far exceed lightspeed $c$. In this appendix we treat a simple model relativistically and show that then $v<c$ for all parameter choices. The model is a circular ring of radius $a$ with mass $m$ and charge $q$ uniformly distributed around the ring. Let the ring lie in the $x-y$ plane and be constrained to rotate about the $z$-axis, its symmetry axis, with angular velocity $z$-component $\omega_{z}=\dot{\alpha}$, where $\alpha(t)$ is the relevant Euler angle. If one neglects/ignores the self magnetic field and there are no applied fields, the nonrelativistic expression for the $z$-component of the canonical spin angular momentum is simply $S_{z}=m a^{2} \dot{\alpha}$. If this expression must have magnitude $\hbar / 2$, one obtains $\beta=|\dot{\alpha}| a / c=\hbar / 2 m a c$. Using the electron mass $m \approx 10^{-27} \mathrm{gm}$ and radius $a \leq 10^{-5} \mathrm{fm}$ yields $\beta \geq 10^{7}$ !

Evidently we should have used relativistic expressions for the momenta. For this model rotator, any infinitesimal ring segment of restmass $d m$ has tangential linear momentum $\gamma a \dot{\alpha} d m$, where $\gamma=\left(1-a^{2} \dot{\alpha}^{2} / c^{2}\right)^{-1 / 2}$. Therefore, in the absence of magnetic fields the spin angular momentum of the ring is $S_{z}=m \gamma a^{2} \dot{\alpha}$. For $\left|S_{z}\right|=\hbar / 2$, one obtains $\beta \gamma=\hbar / 2 m a c \equiv \Lambda$, whereby $\beta=\Lambda / \sqrt{1+\Lambda^{2}}<1$. For the electron parameter values above, $\Lambda \geq 10^{7}$, whereby $\beta \geq 1-0.5 \times 10^{-14}$ ! For baryon parameter values, $m \geq 1.8 \times 10^{-24} \mathrm{gm}, a \geq 1 \mathrm{fm}$, one obtains $\beta \leq 0.1$, which is essentially nonrelativistic.

It is interesting to to evaluate the magnitude $\mu$ of the magnetic moment predicted by this electron model due to its intrinsic rotation only. Since $\beta \approx 1$, $\mu \approx e a / 2=e \hbar / 2 m c \Lambda$, at most $10^{-7}$ times the correct value. This result supports the old idea that the electron magnetic moment may be almost entirely due to highly irregular relativistic translational motion (zitterbewegung) that could be induced by a stochastic field such as the SZPF.

\section{Scientific Research Publishing}

Submit or recommend next manuscript to SCIRP and we will provide best service for you:

Accepting pre-submission inquiries through Email, Facebook, LinkedIn, Twitter, etc. A wide selection of journals (inclusive of 9 subjects, more than 200 journals)

Providing 24-hour high-quality service

User-friendly online submission system

Fair and swift peer-review system

Efficient typesetting and proofreading procedure

Display of the result of downloads and visits, as well as the number of cited articles

Maximum dissemination of your research work

Submit your manuscript at: http://papersubmission.scirp.org/

Or contact jmp@scirp.org 